\title{
Smith's Theorem and a characterization of the 6-cube as distance-transitive graph
}

\author{
M. R. Alfuraidan · J. I. Hall
}

Received: 20 July 2005 / Accepted: 20 January 2006 / Published online: 11 July 2006

(C) Springer Science + Business Media, LLC 2006

\begin{abstract}
A generic distance-regular graph is primitive of diameter at least two and valency at least three. We give a version of Derek Smith's famous theorem for reducing the classification of distance-regular graphs to that of primitive graphs. There are twelve cases - the generic case, four canonical imprimitive cases that reduce to the generic case, and seven exceptional cases. All distance-transitive graphs were previously known in six of the seven exceptional cases. We prove that the 6-cube is the only distance-transitive graph coming under the remaining exceptional case.
\end{abstract}

Keywords Imprimitive distance-transitive graph · Imprimitive distance-regular graph

\section{Introduction}

An important theorem by Derek Smith [14] is frequently cited as the avenue for reduction of the classification of distance-regular and distance-transitive graphs to that of primitive graphs. Occasionally these citations (and proofs) are inexact and the specific reduction not clear. It is our view that a generic distance-regular graph is primitive of diameter at least two and valency at least three. In Theorem 2.9 below we give a version of Smith's Theorem which describes precisely how most distanceregular graphs can be reduced to the generic case and details the exceptions.

M. R. Alfuraidan $(\bowtie)$

Department of Mathematical Sciences, King Fahd University of Petroleum and Minerals, Dhahran 31261, Saudi Arabia

e-mail: monther@kfupm.edu.sa

\section{J. I. Hall}

Department of Mathematics, Michigan State University, East Lansing, Michigan 48824, USA

e-mail: jhall@math.msu.edu 
Brouwer, Cohen, and Neumaier [5, p. 416] have conjectured that one case, that of antipodal and bipartite distance-regular graphs of diameter 6 and valency at least 3 , leads uniquely to the 6-cube. In Theorem 3.3 we prove their conjecture for the class of distance-transitive graphs.

In Section 2 we give an introduction to imprimitivity and reduction for distanceregular graphs, and we present several versions of Smith's Theorem. In the most detailed version (Theorem 2.9) there are twelve cases-the generic primitive case, four canonical imprimitive cases that reduce to the generic case, and seven exceptional cases. In Section 3 we discuss this case division in the context of distancetransitive graphs. In six of the exceptional cases all distance-transitive examples have been characterized previously, and we provide here the classification in the remaining exceptional case.

All graphs and groups that we consider are finite. We usually blur the distinction between a graph and its set of vertices. Our general reference is the book of Brouwer, Cohen, and Neumaier [5].

\section{Smith's Theorem}

Derek Smith [14] observed that the possible blocks of imprimitivity for a distancetransitive graph $(D T G)$ are very limited, and it was soon noted that (with the appropriate definitions) this remains true for distance-regular graphs (DRGs). We present a detailed version of Smith's result. While nothing in this section is strictly new, it is difficult to find in the literature a precise version of Smith's Theorem with complete proof. We make clear the case divisions involved in using Smith's Theorem inductively to classify all distance-regular and distance-transitive graphs. For related refinements of Smith's Theorem, see [3] and [5, p. 140].

If the distance-regular graph $\Gamma$ is disconnected, then each connected component is itself a distance-regular graph. Different components have the same size and intersection numbers. (Different components need not be isomorphic, although they are if $\Gamma$ is distance-transitive.) Therefore we usually restrict our attention to connected distance-regular graphs. We write $k$ for $p_{11}^{0}$, the valency of $\Gamma$, and also $\lambda=p_{11}^{1}$ and $\mu=p_{11}^{2}$. (Recall that if vertices $a$ and $b$ are at distance $h$ in $\Gamma$, then the intersection number $p_{i j}^{h}$ is the number of vertices $z$ at distance $i$ from $a$ and distance $j$ from $b$.)

The connected components of a graph are special examples of congruence classes of the graph. Let $\Gamma$ be a distance-regular graph of diameter $d$. A subset $I \subseteq[0, d]$ is a congruence of $\Gamma$ if $\bigcup_{i \in I} \Gamma^{i}$ is an equivalence relation on $\Gamma$, where the relation $\Gamma^{i}$ consists of the pairs of vertices at distance $i$ in $\Gamma$. Clearly 0 must be in $I$ and any union is symmetric, so transitivity is the only issue.

Associated with any congruence $I$ there are two types of related graphs-the fibers, that is, the equivalence classes, and the quotient $\Gamma / I$, whose vertices are the fibers with two adjecent if they contain adjacent vertices of $\Gamma$. There are two trivial congruences, namely $I=[0]$ where $\Gamma / I=\Gamma$ and $I=[0, d]$ where $\Gamma$ itself is the only fiber. If there are nontrivial congruences, then we call $\Gamma$ imprimitive.

If $\Gamma$ is a distance-transitive graph, then imprimitivity corresponds precisely to imprimitivity for the action of $\operatorname{Aut}(\Gamma)$ on $\Gamma$, with the fibers being the blocks of the 
associated system of imprimitivity. As with the group case, one often focuses on primitive distance-regular graphs, since any $D R G$ can be decomposed (in some sense) into primitive $D R G$ s by passing to fibers and quotients.

A graph is bipartite if it can be partitioned into two nonempty parts so that all edges pass from one part to the other; in particular $\{0,2,4, \ldots\}$ is a congruence, each fiber being within one part or the other. A graph of diameter $d$ is antipodal if $\{0, d\}$ is a congruence.

The original result is

Theorem 2.1 (Smith [14]). Let $\Gamma$ be a connected distance-regular graph with valency $k \geq 3$. If $\Gamma$ is imprimitive, it is either bipartite or antipodal. Both possibilities can occur in the same graph.

For instance, the complete bipartite graph $K_{k, k}$ is both bipartite and antipodal. A connected graph of valency 2 is an $n$-cycle $C_{n}$ and has blocks of imprimitivity of size $m$ for all proper divisors $m$ of $n$. In particular $C_{9}$ is imprimitive but neither bipartite nor antipodal.

For the nonnegative integers $t$ and $d$, we let $\langle t\rangle=\langle t\rangle_{d}$ be the set of all multiples of $t$ that lie in the interval $[0, d]$. Therefore a distance-regular graph $\Gamma$ of diameter $d$ that is bipartite has $\langle 2\rangle$ as a congruence, while $\Gamma$ is antipodal precisely when $\langle d\rangle$ is a congruence. The trivial congruences are $\langle 0\rangle$ and $\langle 1\rangle$.

Theorem 2.2. Let $\Gamma$ be a connected distance-regular graph of diameter $d$, and let I be a congruence of $\Gamma$.

1. There is a $t$ with $I=\langle t\rangle$.

2. If $2<t<d$, then $\Gamma$ has valency 2 .

3. If $t=2$ then $\Gamma$ either is a regular, complete multipartite graph $K_{r, \ldots, r}$ (of diameter 2) or is bipartite.

\section{Proof:}

(1) By transitivity, if $p_{i j}^{h} \neq 0$ with $i, j \in I$ then $h \in I$ as well. Therefore:

(a) If $t \in I$, then $\langle t\rangle \subseteq I$.

(b) If $i, j \in I$ with $i<j$, then $j-i \in I$.

Assume that we do not have $I=\langle 0\rangle$, and let $t$ be the smallest nonzero member of $I$. By $(a)$ we have $I \supseteq\langle t\rangle$. Indeed by $(b)$ we have equality.

(2) Now assume that $0<t<d$ and the valency $k$ is at least 3 . We wish to prove that $t \leq 2$.

Let $\left\{g_{0}, g_{1}, \ldots, g_{t+1}\right\}$ be a path in $\Gamma$ connecting the vertices $g_{0}, g_{t+1}$ at distance $t+1 \leq d$. Consider an arbitrary vertex $g$ adjacent to $g_{t}$ but not equal to $g_{t+1}$. Thus $g_{0}$ and $g$ are at distance $t-1, t$, or $t+1$. If that distance is $t$ then $\left\{g_{0}, g_{t}, g\right\}$ exhibits $p_{t t}^{1} \neq 0$, so $t=1$. If that distance is $t+1$ then $\left\{g_{1}, g_{t+1}, g\right\}$ exhibits $p_{t t}^{1}+p_{t t}^{2} \neq 0$, hence $t \leq 2$. So we may assume that any such $g$ is at distance $t-1$ from $g_{0}$. That is, $p_{1, t-1}^{t}=k-1$. In particular, since $g_{1}$ and $g_{t+1}$ are at distance $t$, there are $k-1$ vertices at distance $t-1$ from $g_{1}$ and adjacent to $g_{t+1}$. Any such vertex must be at distance $t$ from $g_{0}$, one example being $g_{t}$. As $k-1 \geq 2$, there is an $f \neq g_{t}$ at 
distance $t-1$ from $g_{1}$ and adjacent to $g_{t+1}$. But then $\left\{g_{0}, g_{t}, f\right\}$ gives $p_{t t}^{2} \neq 0$. Thus in all cases $t \leq 2$.

(3) Assume that $\langle 2\rangle$ is a congruence but $\Gamma$ is not bipartite. If $\Gamma$ has no triangles, then by hopping around an odd circuit we could discover that adjacent vertices are in the same fiber for $\langle 2\rangle$, which is not the case. Therefore $\Gamma$ contains triangles.

Suppose $\left\{g_{0}, g_{1}, g_{2}, g_{3}\right\}$ is an induced path in $\Gamma$, and let $\left\{g, g_{0}, g_{1}\right\}$ be a triangle. The vertex $g_{2}$ is not adjacent to $g_{0}$ by assumption, so it is adjacent to $g$ as $p_{22}^{1}=0$. But similarly the vertex $g_{3}$, not adjacent to $g_{1}$, must also be adjacent to $g$. Thus $g_{0}, g, g_{3}$ is a path of length 2 , and we conclude that $\Gamma$ has diameter 2 . Nonadjacency is now the equivalence relation $\langle 2\rangle$, so $\Gamma$ is complete multipartite. As it also is regular, each part has fixed size $r$, say.

In particular, a fiber is always a connected component under some relation $\Gamma^{t}$.

Let $\Gamma$ be a connected $D R G$ of diameter $d$. If $\langle d\rangle$ is a nontrivial congruence, then we say that $\Gamma$ is antipodal imprimitive (usually shortened to antipodal, although this is a mild abuse since complete graphs are antipodal and primitive). Similarly $\Gamma$ is even imprimitive (or even) if $\langle 2\rangle$ is a nontrivial congruence.

Theorem 2.2 shows that a connected, imprimitive $D R G$ of valency at least 3 is either antipodal or even and that, when even, it is either bipartite or complete multipartite (and hence antipodal of diameter 2). In particular, this gives a proof of Smith's Theorem 2.1. We now want to use our precise knowledge of congruences to decompose every $D R G$ into primitive pieces.

In the bipartite case, the two fibers or halves are uniquely determined (by Theorem 2.2 ) as the only connected components under the adjacency relation $\approx$ given by having distance 2 in $\Gamma$. We write $\mathrm{B} \Gamma$ for either one of the halves. This is somewhat ambiguous since the two graphs $(\mathrm{B} \Gamma, \approx)$ need not be isomorphic, but the abuse is usually not a problem. The two graphs have the same intersection numbers (in particular, the same number of vertices), and in a $D T G$ they are indeed isomorphic. When appropriate we write $\mathrm{B}^{-} \Gamma$ and $\mathrm{B}^{+} \Gamma$ to distinguish the two halves. The graph $(\mathrm{B} \Gamma, \approx)$ is a halved graph of $\Gamma$, and $\Gamma$ is a doubled graph (or doubling) of $\mathrm{B} \Gamma$. Similarly we write $\mathrm{A} \Gamma$ for the quotient graph induced on the fibers of an antipodal graph $\Gamma$. We then say that $\Gamma$ is an antipodal cover of $\mathrm{A} \Gamma$ and even an antipodal $r$-cover, where $r$ is the common cardinality of the antipodal classes. The antipodal quotient $\mathrm{A} \Gamma$ is also called a folded graph.

We have two elementary but fundamental observations; see [5, Proposition 4.2.2], $[3,14]$.

Proposition 2.3. Let $\Gamma$ be a connected, bipartite DRG of valency $k$ and diameter $d(>1)$. Then $(\mathrm{B} \Gamma, \approx)$ is a connected DRG of valency $k(k-1) / \mu$ and diameter $\lfloor d / 2\rfloor$. For $0 \leq i \leq\lfloor d / 2\rfloor$, two vertices are at distance $i$ in $\mathrm{B} \Gamma$ if and only if they are at distance $2 i$ in $\Gamma$. If $\Gamma$ is a DTG, then $\mathrm{B} \Gamma$ is also a DTG.

If the set of $\Gamma$-relations $I \subseteq\langle 2\rangle_{d}$ gives a congruence of $\left(\mathrm{B}^{\epsilon} \Gamma, \approx\right)$, then I also gives a congruence of $\left(\mathrm{B}^{-\epsilon} \Gamma, \approx\right)$ and of $\Gamma$ itself.

Proposition 2.4. Let $\Gamma$ be a connected, antipodal DRG of valency $k$ and diameter $d(>1)$. Then $\mathrm{A} \Gamma$ is a connected DRG of diameter $\lfloor d / 2\rfloor$. For $0 \leq i \leq\lfloor d / 2\rfloor$, two Springer 
vertices (fibers) $\alpha$ and $\beta$ are at distance $i$ in $\mathrm{A} \Gamma$ if and only if each pair $a \in \alpha$ and $b \in \beta$ is at distance $i$ or $d-i$ in $\Gamma$. If $d \geq 3$ then $\mathrm{A} \Gamma$ also has valency $k$. If $\Gamma$ is a $D T G$, then $\mathrm{A} \Gamma$ is also a DTG.

A symmetric design is an incidence system $\mathcal{D}$ of $v$ points and $v$ blocks, with $k$ points per block and $k$ blocks per point, and with the additional condition that each pair of distinct blocks intersects in exactly $\mu$ points, the intersection index, and dually each pair of points is together in exactly $\mu$ blocks. The design is nontrivial if $1<k<v$ or, equivalently, if $0<\mu<k$. (See [5, p. 439] or [12].)

Proposition 2.5. Let $\Gamma$ be a connected distance-regular graph of diameter $d$ and valency $k$ in which $\langle 2\rangle$ is a congruence. Then one of:

1. $0 \leq d \leq 1$ or $0 \leq k \leq 2$; that is, $\Gamma$ is $K_{k+1}$ or $C_{n}$, for some $n$;

2. $d=2$, and $\Gamma$ is a regular, complete multipartite graph;

3. $d=3$, and $\Gamma$ is the bipartite incidence graph of a nontrivial symmetric design with block size $k \geq 3$ and index $\mu$;

4. $d=4$; $\Gamma$ is antipodal and bipartite; $\mathrm{A} \Gamma$ is $K_{k, k}$ with $k \geq 3$, and $\mathrm{B} \Gamma$ is complete multipartite;

5. $d \geq 4 ; \Gamma$ is bipartite; $\mathrm{B} \Gamma$ has diameter $\lfloor d / 2\rfloor \geq 2$ and valency $k_{0}=k(k-1) / \mu \geq$ $k \geq 3$, and $\mathrm{B} \Gamma$ is not even.

Proof: Assume we are not in the first case, so $d \geq 2$ and $k \geq 3$. If $d=2$, then we have the second case by Theorem 2.2.3. (A bipartite graph of diameter 2 is complete.)

Thus we may assume that $d \geq 3$, so by Theorem 2.2.3 the graph $\Gamma$ is bipartite (with each half connected under $\approx$ ). Furthermore by Proposition 2.3 the halved graphs $\mathrm{B} \Gamma$ have diameter $\lfloor d / 2\rfloor$ and valency $k_{0}=k(k-1) / \mu$. If this is less than $k$, then $\mu>k-1$ hence $\mu=k$; but then $\Gamma$ is $K_{k, k}$ of diameter 2 , which is not the case. Thus $k_{0} \geq k$.

Suppose $d=3$. Let the two halves В $\Gamma$ be $P$ (points) and $B$ (blocks). Each block is incident to (adjacent to) $k$ points, and dually each point is incident to $k$ blocks. As each half $\mathrm{B} \Gamma$ is complete under $\approx$, each pair of points is incident to exactly $\mu$ common blocks, and each pair of blocks is incident to exactly $\mu$ common points. Finally $0<\mu<k$, since $\Gamma$ has diameter 3 . This gives the third conclusion.

It remains to consider the case where $d \geq 4$. Here $\Gamma$ is bipartite with $\mathrm{B} \Gamma$ of diameter $\lfloor d / 2\rfloor \geq 2$ and valency $k_{0} \geq k \geq 3$. When $\mathrm{B} \Gamma$ is even, $\langle 4\rangle$ is a congruence of $\Gamma$. As $d \geq 4$ and $k \geq 3$, we then have $d=4$ by Theorem 2.2. That is, $\Gamma$ is also antipodal of diameter 4 . This implies that $\mathrm{A} \Gamma$ is bipartite of diameter 2, hence complete bipartite. Also $\mathrm{B} \Gamma$ is antipodal of diameter 2 and so is complete multipartite by Theorem 2.2.3.

Remark. Under Proposition 2.5.3 the converse is also true: the incidence graph of a nontrivial symmetric design is a bipartite $D R G$ of diameter 3. (The block size is 2 if and only if the incidence graph is $C_{6}$.) The relationship between bipartite DRGs of diameter 3 and symmetric designs was noted by Hemmeter [8, 9]. 
Proposition 2.6. Let $\Gamma$ be a connected distance-regular graph of diameter $d$ and valency $k$ in which $\langle d\rangle$ is a congruence. Then one of:

1. $0 \leq d \leq 1$ or $0 \leq k \leq 2$; that is, $\Gamma$ is $K_{k+1}$ or $C_{n}$, for some $n$;

2. $d=2$, and $\Gamma$ is a regular, complete multipartite graph;

3. $d=3$, and $\Gamma$ is an antipodal cover of $K_{k+1}$ with $k \geq 3$;

4. $d=4 ; \Gamma$ is antipodal and bipartite; $\mathrm{A} \Gamma$ is $K_{k, k}$ with $k \geq 3$, and $\mathrm{B} \Gamma$ is complete multipartite;

5. $d \geq 4$; $\mathrm{A} \Gamma$ has diameter $\lfloor d / 2\rfloor \geq 2$ and valency $k \geq 3$, and $\mathrm{A} \Gamma$ is not antipodal.

Proof: Assume that we do not have the first two cases, so $d \geq 3$ and $k \geq 3$ as in the previous proposition. If $d=3$ then $\mathrm{A} \Gamma$ has diameter $\lfloor 3 / 2\rfloor=1$ and valency $k$ by Proposition 2.4, giving the third conclusion.

Now let $d \geq 4$, so that $\mathrm{A} \Gamma$ has diameter $e=\lfloor d / 2\rfloor \geq 2$ and valency $k$ (again by Proposition 2.4). Suppose that $\mathrm{A} \Gamma$ is antipodal. Then $I=\{0, e, d-e, d\}$ is a congruence of $\Gamma$ with $|(d-e)-e| \leq 1$. Therefore by Theorem 2.2 we have $d=4=$ $2 e$, and $\Gamma$ is both bipartite and antipodal of diameter 4 . As before, we have conclusion (4).

Lemma 2.7. Let $\Gamma$ be a connected DRG of diameter $d=2 e+1 \geq 5$ and valency $k \geq 3$.

1. If $\Gamma$ is antipodal, then $\mathrm{A} \Gamma$ is primitive.

2. If $\Gamma$ is bipartite, then $\mathrm{B} \Gamma$ is primitive.

Proof: If $\Gamma$ is antipodal, then by Proposition 2.6 the quotient $\mathrm{A} \Gamma$ is not antipodal. As there are paths in $\Gamma$ connecting vertices at distance $d=2 e+1$, the quotient $\mathrm{A} \Gamma$ has odd circuits and so is not bipartite. Thus $\mathrm{A} \Gamma$ is primitive by Smith's Theorem 2.1.

If $\Gamma$ is bipartite, then by Proposition 2.5 the graph $\mathrm{B} \Gamma$ is not bipartite. If $\mathrm{B} \Gamma$ is antipodal, then $\{0,2 e\}$ is a congruence of $\mathrm{B} \Gamma$ and $\Gamma$ by Proposition 2.3, which is not the case since $2 e \neq d \geq 5$ and $k \geq 3$.

Lemma 2.8. Let $\Gamma$ be a connected DRG of diameter $d=2 e \geq 4$ and valency $k \geq 3$.

1. Assume $\Gamma$ is antipodal. Then $\mathrm{A} \Gamma$ is even if and only if $\Gamma$ is bipartite, in which case $\mathrm{A} \Gamma$ is bipartite.

2. Assume $\Gamma$ is bipartite. Then $\mathrm{B} \Gamma$ is antipodal if and only if $\Gamma$ is antipodal.

3. Assume $\Gamma$ is bipartite and antipodal. Then we have $\left\{\mathrm{B}^{-} \mathrm{A} \Gamma, \mathrm{B}^{+} \mathrm{A} \Gamma\right\}=$ $\left\{\mathrm{AB}^{-} \Gamma, \mathrm{AB}^{+} \Gamma\right\}$.

Proof: Assume $\Gamma$ is antipodal. By Proposition 2.4 the fibers or vertices of $\mathrm{A} \Gamma$ are the $\{0, d\}$-classes with two such vertices at distance $i$, for $0 \leq i \leq e$, when the corresponding fibers have relation $\{i, 2 e-i\}$. So $\mathrm{A} \Gamma$ is even precisely when $I=\cup_{j}\{2 j, 2 e-2 j\}$ is a congruence of $\mathrm{A} \Gamma$ and so of $\Gamma$. This is equivalent to $\Gamma$ itself being even and so bipartite by Theorem 2.2.3.

The even graph $\mathrm{A} \Gamma$ is bipartite or complete multipartite. If $\mathrm{A} \Gamma$ is complete multipartite, then it is antipodal; so by Proposition 2.6 A $\Gamma$ is $K_{k, k}$ and again bipartite. The 祭Springer 
$D R G$ s $\mathrm{B}^{\epsilon} \mathrm{A} \Gamma$ have as vertices the $\{0, d\}$-classes, two such adjacent when they are in relation $\{2, d-2\}$.

Assume $\Gamma$ is bipartite. Within the $D R G$ s $\left(\mathrm{B}^{\epsilon} \Gamma, \approx\right)$, the distances $0,1, \ldots, e$ are given by the $\Gamma$-distances $0,2, \ldots, 2 e$. By Proposition $2.3, \mathrm{~B}^{\epsilon} \Gamma$ is antipodal if and only if $\{0,2 e\}$ is a congruence of $\mathrm{B}^{-} \Gamma, \mathrm{B}^{+} \Gamma$, and $\Gamma$ itself. The $D R G \mathrm{~s} \mathrm{AB}^{\epsilon} \Gamma$ then have as vertices the $\{0, d\}$-classes with adjacency given by the relation $\{2, d-2\}$.

When $\Gamma$ is both antipodal and bipartite, we see that the graphs $\mathrm{B}^{\epsilon} \mathrm{A} \Gamma$ and $\mathrm{AB}^{\epsilon} \Gamma$ have the same vertices and adjacency relation, giving (3).

We now revisit Smith's Theorem.

Theorem 2.9. Let $\Gamma$ be a connected distance-regular graph of diameter $d$ and valency $k$. Set $\mu=p_{11}^{2}$ and $k_{0}=k(k-1) / \mu$. Then one of:

1. $\Gamma$ is primitive of diameter $d \geq 2$ and valency $k \geq 3$;

2. $k=2, d=\lfloor n / 2\rfloor$, and $\Gamma$ is a cycle $C_{n}$ for some $n \geq 3$;

3. $d \leq 1$, and $\Gamma$ is a complete graph $K_{k+1}$;

4. $d=2$, and $\Gamma$ is a complete multipartite graph $K_{r, \ldots, r}$ with $1+\frac{k}{r}$ parts of size $r \geq 2$

5. $d=3$, and $\Gamma$ is the bipartite incidence graph of a nontrivial symmetric design with block size $k \geq 3$ and index $\mu$;

6. $d=3$, and $\Gamma$ is an antipodal cover of $K_{k+1}$ with $k \geq 3$;

7. $d=4 ; \Gamma$ is antipodal and bipartite; $\mathrm{A} \Gamma$ is $K_{k, k}$ with $k \geq 3$, and $\mathrm{B} \Gamma$ is complete multipartite;

8. $d=6 ; \Gamma$ is antipodal and bipartite; $\mathrm{A} \Gamma$ is bipartite of diameter 3 , and $\mathrm{B} \Gamma$ is antipodal of diameter 3 ; the graphs $\left\{\mathrm{B}^{-} \mathrm{A} \Gamma, \mathrm{B}^{+} \mathrm{A} \Gamma\right\}=\left\{\mathrm{AB}^{-} \Gamma, \mathrm{AB}^{+} \Gamma\right\}$ are $K_{k_{0}+1}$, for $k_{0} \geq k \geq 3$;

9. $d \geq 4 ; \Gamma$ is antipodal but not bipartite, and $\mathrm{A} \Gamma$ is primitive of diameter $\lfloor d / 2\rfloor \geq 2$ and valency $k \geq 3$;

10. $d \geq 4 ; \Gamma$ is bipartite but not antipodal, and $\mathrm{B} \Gamma$ is primitive of diameter $\lfloor d / 2\rfloor \geq 2$ and valency $k_{0} \geq k \geq 3$;

11. odd $d=2 e+1 \geq 5 ; \Gamma$ is antipodal and bipartite; all antipodal classes have size 2 , and $\mathrm{A} \Gamma$ is primitive of diameter $e \geq 2$ and valency $k \geq 3$; $\mathrm{B} \Gamma$ is primitive of diameter $e \geq 2$ and valency $k_{0} \geq k \geq 3$;

12. even $d=2 e \geq 8 ; \Gamma$ is antipodal and bipartite; $\mathrm{A} \Gamma$ is bipartite of diameter $e$, and $\mathrm{B} \Gamma$ is antipodal of diameter ; the graphs $\left\{\mathrm{B}^{-} \mathrm{A} \Gamma, \mathrm{B}^{+} \mathrm{A} \Gamma\right\}=\left\{\mathrm{AB}^{-} \Gamma, \mathrm{AB}^{+} \Gamma\right\}$ are primitive of diameter $\lfloor e / 2\rfloor \geq 2$ and valency $k_{0} \geq k \geq 3$.

Proof: Assume that we do not have (1), (2), or (3). Therefore $\Gamma$ is imprimitive of diameter $d \geq 2$ and valency $k \geq 3$. If $d$ is 2 or 3 , then we have one of (4), (5), (6) by Theorem 2.2 and Propositions 2.5 and 2.6. We now assume that $d \geq 4$.

First suppose that $\Gamma$ is antipodal but not bipartite. By Proposition 2.6, А $\Gamma$ has diameter $\lfloor d / 2\rfloor \geq 2$, valency $k \geq 3$, and is not antipodal. If $\mathrm{A} \Gamma$ is not primitive, then it is bipartite and by Lemma 2.7.1 its diameter would be even; but then $\Gamma$ would be bipartite by Lemma 2.8.1, against assumption. This gives case (9).

Next suppose that $\Gamma$ is bipartite but not antipodal. By Propostion 2.5, В $\Gamma$ has diameter $\lfloor d / 2\rfloor \geq 2$, valency $k_{0} \geq k \geq 3$, and is not even. If $\mathrm{B} \Gamma$ is not primitive, then 
it is antipodal and by Lemma 2.7.2 its diameter would be even. Then $\Gamma$ itself would be antipodal by Lemma 2.8.2, a contradiction. This gives case (10).

Therefore we may assume for the balance of the proof that $\Gamma$ is both antipodal and bipartite. We have $\mathrm{A} \Gamma$ and $\mathrm{B} \Gamma$ of diameter $e=\lfloor d / 2\rfloor$ and of respective valencies $k(\geq 3)$ and $k_{0}(\geq k \geq 3)$ by Propositions 2.5 and 2.6.

For odd $d=2 e+1 \geq 5$, by Lemma 2.7 both $\mathrm{A} \Gamma$ and $\mathrm{B} \Gamma$ are primitive. If the antipodal classes had size greater than 3 , then there would be cycles of odd length $3 d$ in bipartite $\Gamma$, which does not happen. This gives case (11).

Now we additionally assume $d=2 e \geq 4$. By Lemma 2.8.1-2, $\mathrm{A} \Gamma$ is bipartite and $\mathrm{B} \Gamma$ is antipodal. By Lemma 2.8.3, the $D R G \mathrm{~s}\left\{\mathrm{~B}^{-} \mathrm{A} \Gamma, \mathrm{B}^{+} \mathrm{A} \Gamma\right\}=\left\{\mathrm{AB}^{-} \Gamma, \mathrm{AB}^{+} \Gamma\right\}$ have diameter $\lfloor e / 2\rfloor$ and valency $k_{0} \geq k \geq 3$.

If $d=4$, then we have case (7) by Propositions 2.5 and 2.6. If $d=6$, then the $D R G \mathrm{~s}\left\{\mathrm{~B}^{-} \mathrm{A} \Gamma, \mathrm{B}^{+} \mathrm{A} \Gamma\right\}=\left\{\mathrm{AB}^{-} \Gamma, \mathrm{AB}^{+} \Gamma\right\}$ have diameter $1=\lfloor 3 / 2\rfloor$; they are complete, giving case (8).

Now assume $d=2 e \geq 8$, and choose notation so that $\mathrm{B}^{\epsilon} \mathrm{A} \Gamma=\mathrm{AB}^{\epsilon} \Gamma$. To show that we are in the final case (12), we must prove that the $D R G \mathrm{~s}^{\epsilon} \mathrm{A} \Gamma=\mathrm{AB}^{\epsilon} \Gamma$ are primitive.

Set $\Delta=\mathrm{A} \Gamma$ of diameter $e \geq 4$ and valency $k \geq 3$. By Proposition 2.5 the halved graphs $\mathrm{B}^{\epsilon} \Delta\left(=\mathrm{B}^{\epsilon}(\mathrm{A} \Gamma)\right)$ are not even, except possibly when $e=4$ and $\Delta$ is an antipodal cover of a complete bipartite graph. On the other hand, as $\Gamma$ has diameter at least 8 , its quotient $\Delta=\mathrm{A} \Gamma$ is not antipodal by Proposition 2.6. We conclude that $\mathrm{B}^{\epsilon} \mathrm{A} \Gamma$ is not even.

Next set $\Phi=\mathrm{B}^{\epsilon} \Gamma$ (for a fixed but arbitrary $\epsilon$ ) of diameter $e \geq 4$ and valency $k_{0} \geq 3$. By Proposition 2.6 the quotient graph $\mathrm{A} \Phi\left(=\mathrm{A}\left(\mathrm{B}^{\epsilon} \Gamma\right)\right)$ is not antipodal, except possibly when $e=4$ and $\Phi$ is a bipartite, antipodal cover of a complete bipartite graph. On the other hand, as $\Gamma$ has diameter at least 8 , its halved graph $\Phi=\mathrm{B}^{\epsilon} \Gamma$ is not even by Proposition 2.5. We conclude that $\mathrm{AB}^{\epsilon} \Gamma$ is not antipodal.

We now have that $\mathrm{B}^{\epsilon} \mathrm{A} \Gamma$ is not even and $\mathrm{AB}^{\epsilon} \Gamma$ is not antipodal. Therefore $\mathrm{B}^{\epsilon} \mathrm{A} \Gamma=$ $\mathrm{AB}^{\epsilon} \Gamma$ is primitive as in (12), completing the proof of the theorem.

Corollary 2.10. Let $\Gamma$ be a connected distance-regular graph. Then almost always $\Gamma$ occurs under a unique case in Theorem 2.9. The only exceptions are given by $C_{3}=K_{3}$, which comes under (2.9.2) and (2.9.3); $C_{4}=K_{2,2}$, which comes under (2.9.2) and (2.9.4); and $K_{k+1, k+1} \backslash \mathbf{1}$ (the complete bipartite graph minus a 1-factor), which among valency $k(\geq 3)$ graphs is the unique antipodal graph under (2.9.5) and the unique bipartite graph under (2.9.6).

Proof: Primitive graphs occur under (2.9.1), (2.9.2) (for $n$ prime), and (2.9.3); so $C_{3}=K_{3}$ is the only overlap in the primitive case. The graph $C_{4}=K_{2,2}$ is the only valency 2 graph in (2.9.4-12).

By Propositions 2.5 and 2.6, a $D R G$ of diameter 3 and valency $k(\geq 3)$ is bipartite and antipodal if and only if it occurs under both (2.9.5) and (2.9.6). Suppose an incidence graph as in (2.9.5) comes from a design with $v$ points, $k$ points per block, and $v-k>1$. Then for a block $b$ not incident to points $p_{1}, p_{2}$, the block $b$ has distance 3 from both $p_{1}$ and $p_{2}$, which are at distance 2 from each other. Thus this graph is not antipodal. Therefore a graph under both (2.9.5) and (2.9.6) must be the incidence 
graph of a symmetric design with $k+1$ points and $k$ points per block. Such a graph is a complete bipartite graph $K_{k+1, k+1}$ minus a 1-factor (a matching).

The graphs of (2.9.7), (2.9.8), (2.9.11), and (2.9.12) are distingished from each other by their diameters and from (2.9.9) and (2.9.10) by having imprimitive antipodal quotients and halved graphs. Clearly no graph comes under both (2.9.9) and (2.9.10).

\section{A characterization of the 6-cube}

Consider the various cases of Theorem 2.9 when we restrict our attention to distancetransitive graphs $\Gamma$.

Due to the efforts of many people, the complete classification of distance-transitive graphs in the generic primitive case (2.9.1) is nearly complete; see, for instance, [10] and [13].

A large segment of the four canonical imprimitive distance-transitive cases (2.9.912) has also been handled; see [1, 2, 4, 8, 9].

For the exceptional cases, the specific graphs of (2.9.2-4) are all distance-transitive. The classification of distance-transitive antipodal covers of complete graphs, as in (2.9.6), and of complete bipartite graphs, as in (2.9.7), has been completely settled by, respectively, Godsil, Liebler, and Praeger in [7] and Ivanov, Liebler, Penttila, and Praeger in [11].

It remains to discuss the exceptional cases (2.9.5) and (2.9.8).

The bipartite, diameter 3 distance-regular graphs of (2.9.5) are exactly the incidence graphs of nontrivial symmetric designs. It is routine to extend this to

Proposition 3.1. The distance-transitive graph $\Gamma$ is bipartite of diameter 3 if and only if it is the incidence graph of a self-dual nontrivial symmetric design $\mathcal{D}$ for which $\operatorname{Aut}(\mathcal{D})$ is 2-transitive on points.

Given a symmetric design $\mathcal{D}$ with $v$ points, block size $k$, and intersection index $\mu$, its complementary design $\mathcal{D}^{\prime}$ is the symmetric design with the same point set but whose blocks are the complements of the blocks in the original design. Therefore $v$ remains fixed but the complementary design $\mathcal{D}^{\prime}$ has block size $k^{\prime}=v-k$ and index $\mu^{\prime}=v-2 k+\mu$. Clearly $\mathcal{D}$ admits a 2-transitive group of automorphisms if and only if $\mathcal{D}^{\prime}$ does. Since nontrivial symmetric designs are those with $1<k<v$, the complementary design of a nontrivial design is also nontrivial provided $k \neq v-1$. When $k=v-1$ we have the symmetric design of all $k$-subsets of a $(k+1)$-set with $\mu=k-1$. (See Corollary 2.10.)

In view of Proposition 3.1, the classification of DTGs as in (2.9.5) is given by

Theorem 3.2 (Kantor[12]). Let $\mathcal{D}$ be a symmetric design with $v$ points, $k$ points per block, and intersection index $\mu, 3 \leq k<v$. Further assume that $A \leq \operatorname{Aut}(\mathcal{D})$ is 2transitive on the points of $\mathcal{D}$. Then one of: 
1. $\mathcal{D}$ is a Desarguesian projective space with $v=\left(q^{d}-1\right) /(q-1), \quad k=$ $\left(q^{d-1}-1\right) /(q-1)$, and $\mu=\left(q^{d-2}-1\right) /(q-1)$, or its complementary design; $\operatorname{PSL}(d, q) \leq A \leq P \Gamma L(d, q)$ with $d \geq 3$ or $A=\operatorname{Alt}(7)$ and $(d, q)=(4,2)$;

2. $\mathcal{D}$ is the unique Hadamard design with $v=11, k=5$, and $\mu=2$, or its complementary design; $P S L(2,11) \leq A \leq P G L(2,11)$;

3. $\mathcal{D}$ is a unique design with $v=176, k=50$, and $\mu=14$, or its complementary design; $A=H S$, the Higman-Sims group;

4. $\mathcal{D}$ is a design with $v=2^{2 m}, k=2^{m-1}\left(2^{m}-1\right)$, and $\mu=2^{m-1}\left(2^{m-1}-1\right)$, or its complementary design; there are unique 2-transitive, complementary examples for each $m \geq 2 ; A^{(\infty)}$ is $V . A_{0}$ with $V=\mathbb{F}_{2}^{2 m}$ and $A_{0}$ equal to $\operatorname{Sp}\left(2 k, 2^{e}\right)^{\prime}$ for $m=k e$ ( but $(k, e) \neq(1,1)$ ) or $G_{2}\left(2^{e}\right)^{\prime}$ for $m=3 e$.

5. $\mathcal{D}$ is the unique design with $v=k+1$ and $\mu=k-1$; $A$ is any 2-transitive subgroup of $\operatorname{Sym}(k+1)$.

Each of these designs is self-dual. In particular, the incidence graph of each of these designs is a bipartite DTG of diameter 3. Conversely, every bipartite DTG of diameter 3 is one of these incidence graphs.

Here the subgroup $G^{(\infty)}$ denotes the last term in the derived series of the group $G$.

The rest of this section is devoted to the classification of those DTGs that appear under the remaining exceptional case (2.9.8):

Theorem 3.3. An antipodal and bipartite distance-transitive graph of diameter 6 and valency $k \geq 3$ is isomorphic to the 6-cube.

The 6-cube has as vertices the $64=2^{6}$ subsets of a 6 -set with two subsets adjacent when their symmetric difference has size 1 . It is distance-transitive, bipartite, and antipodal (with $r=2$, antipodal fibers consisting of complementary subsets). Brouwer, Cohen, and Neumaier [5, p. 416] have conjectured that the theorem holds under the weaker hypothesis that the graph is distance-regular.

Let $\Gamma$ be a distance-transitive graph as in the theorem: antipodal and bipartite of diameter 6 with valency $k \geq 3$. By Theorem 2.9 we know various additional things about $\Gamma$. Set $k_{0}=k(k-1) / \mu \geq k$ and $v=k_{0}+1$. The graph $\Gamma$ is an antipodal $r$ cover, $r \geq 2$, of the distance-transitive quotient $\mathrm{A} \Gamma$ of diameter 3 , which is the bipartite incidence graph of a symmetric design with $v$ points and $v$ blocks, $k$ points per block, and index $\mu$. The distance-transitive halved graph $\mathrm{B} \Gamma$ is a diameter 3 antipodal $r$-cover of the complete graph $K_{v}$. The primitive graph $\mathrm{BA} \Gamma=\mathrm{AB} \Gamma$ is $K_{v}$.

Lemma 3.4 (Brouwer, Cohen, Neumaier [5], p. 416). We have

$$
(r-1) \mu^{2}(\mu+2)=k \mu+k-\mu .
$$

In particular,

1. $r=\frac{k \mu+k-\mu}{\mu^{2}(\mu+2)}+1$;

2. $\mu$ divides $k$, and $\mu^{2}$ divides $k-\mu$.

Proposition 3.5. We have one of: 
1. $r=(2 q+4) / 3$, and $\mathrm{A} \Gamma$ is the incidence graph of a Desarguesian projective plane of order $q$;

2. $r=2$, and $\mathrm{A} \Gamma$ is the incidence graph of the unique 2-transitive symmetric design with 16 points, 6 points per block, and index 2 .

Proof: The graph $\mathrm{A} \Gamma$ is the incidence graph of one of the symmetric designs $\mathcal{D}$ or $\mathcal{D}^{\prime}$ from Kantor's Theorem 3.2. The possible parameter sets $(v, k, \mu)$ are given in

\begin{tabular}{llll}
\hline Case & $v$ & $k$ & $\mu$ \\
\hline 1 & $\frac{q^{d}-1}{q-1}$ for $d \geq 3$ & $\frac{q^{d-1}-1}{q-1}$ & $\frac{q^{d-2}-1}{q-1}$ \\
$1^{\prime}$ & $\frac{q^{d}-1}{q-1}$ for $d \geq 3$ & $q^{d-1}$ & $q^{d-2}(q-1)$ \\
2 & 11 & 5 & 2 \\
$2^{\prime}$ & 11 & 6 & 3 \\
3 & 176 & 50 & 14 \\
$3^{\prime}$ & 176 & 126 & 90 \\
4 & $2^{2 m}$ for $m \geq 2$ & $2^{m-1}\left(2^{m}-1\right)$ & $2^{m-1}\left(2^{m-1}-1\right)$ \\
$4^{\prime}$ & $2^{2 m}$ for $m \geq 2$ & $2^{m-1}\left(2^{m}+1\right)$ & $2^{m-1}\left(2^{m-1}+1\right)$ \\
5 & $k+1$ for $k \geq 3$ & $k$ & $k-1$ \\
& & & \\
\hline
\end{tabular}

By Lemma 3.4, we must have $k$ divisible by $\mu$. As $\operatorname{gcd}\left(q^{a}-1, q^{a-1}-1\right)=q-1$ and $\operatorname{gcd}\left(2^{m}+1,2^{m-1}+1\right)=1$, that leaves us with

\begin{tabular}{llll}
\hline Case & $v$ & $k$ & $\mu$ \\
1 & $q^{2}+q+1$ & $q+1$ & 1 \\
\hline $1^{\prime}$ & $2^{d}-1$ for $d \geq 3$ & $2^{d-1}$ & $2^{d-2}$ \\
$2^{\prime}$ & 11 & 6 & 3 \\
4 & 16 & 6 & 2 \\
\hline
\end{tabular}

By Lemma 3.4, $\mu^{2}$ divides $k-\mu$. Therefore cases $1^{\prime}$ and $2^{\prime}$ do not occur.

In Case 1

$$
r=\frac{k \mu+k-\mu}{\mu^{2}(\mu+2)}+1=\frac{(q+1) 1+(q+1)-1}{1^{2}(1+2)}+1=\frac{2 q+1}{3}+1 .
$$

In Case 4, Kantor's Theorem 3.2 says that the 2-transitive symmetric design with $(v, k, \mu)=(16,6,2)$ is unique. We have

$$
r=\frac{k \mu+k-\mu}{\mu^{2}(\mu+2)}+1=\frac{6 \cdot 2+6-2}{2^{2}(2+2)}+1=2 .
$$

Lemma 3.6. If $\Gamma$ has parameters $(v, k, \mu, r)=(16,6,2,2)$, then it is the 6-cube.

Proof: The 6-cube has the desired properties. It is relatively well-known that the 6cube is uniquely determined by its parameters [5, p. 416]. Indeed, as such a graph $\Gamma$ 
is bipartite, $\lambda=0$ and

$$
d=6=\frac{2 \cdot 6}{0+2}=\frac{2 k}{\lambda+2}
$$

so [5, Corollary 5.24] applies. As $\lambda=0<1=\mu-1, \Gamma$ is not a Terwilliger graph. Therefore $\Gamma$ is one of four specific graphs of diameter 6 , and of these only the 6 -cube has 64 vertices.

Lemma 3.7. Suppose $\Gamma$ has parameters

$$
(v, k, \mu, r)=\left(q^{2}+q+1, q+1,1,(2 q+4) / 3\right),
$$

and let $H \leq \operatorname{Aut}(\Gamma)$ be distance-transitive on $\Gamma$. Then $H^{(\infty)}$ has a normal elementary abelian 2-subgroup $J$ (possibly trivial) with $H^{(\infty)} / J$ isomorphic to $\operatorname{PSL}(3, q)$.

Proof: Let $K$ be the kernel of the action on the set of antipodal fibers of $\Gamma$. Then $G=H / K$ is distance-transitive on the bipartite incidence graph А $\Gamma$ of a projective plane with parameters $(v, k, \mu)=\left(q^{2}+q+1, q+1,1\right)$. By Kantor's Theorem 3.2, we have $G^{(\infty)}=\operatorname{PSL}(3, q)$.

The kernel $K$ is faithful and semiregular on each fiber, since by connectivity only the identity can fix a vertex and all fibers. If $K \neq 1$, then it is semiregular and normal in the 2-transitive group induced on a given fiber $\alpha$ by its stabilizer $H_{\alpha}$. Thus by Burnside's Theorem [6, Theorem 4.3], $K$ is an elementary abelian $l$-subgroup of order $r$ acting regularly on $\alpha$. Since $r=(2 q+4) / 3$ is even, we have $l=2$.

With $J=K \cap H^{(\infty)}$ we have $H^{(\infty)} / J=G^{(\infty)}$ as described.

Lemma 3.8. $\Gamma$ does not have the parameter set

$$
(v, k, \mu, r)=\left(q^{2}+q+1, q+1,1,(2 q+4) / 3\right) .
$$

Proof: Again let $H$ be a distance-transitive group of automorphisms of $\Gamma$, and consider the group it induces on the halved graph $\mathrm{B} \Gamma$, an antipodal $r$-cover of the complete graph $K_{v}$. The stabilizer $L$ of the two bipartite halves of $\Gamma$ has index 2 in $H$ and is faithful on each half. (As $k>\mu$, the vertex $a$ is the unique vertex adjacent to all the vertices adjacent to $a$. Thus the vertexwise stabilizer of one half also fixes all vertices of the other half.) Therefore $L$ is a distance-transitive group of automorphism of the graph $\mathrm{B} \Gamma$, an antipodal $r$-cover of the complete graph $K_{v}$, and $L$ has index 2 in $H$. By Lemma 3.7, $L$ has a unique nonsolvable composition factor, namely $\operatorname{PSL}(3, q)$.

Godsil, Liebler, and Praeger [7] have classified all distance-transitive antipodal covers of complete graphs and their groups. The group $\operatorname{PSL}(3, q)$ can appear as a composition factor only for $q=2$ (via $\operatorname{PSL}(2,7) \simeq \operatorname{PSL}(3,2)$ ). But this gives $r=$ $(2 \cdot 2+4) / 3=\frac{8}{3}$, a contradiction. Therefore no examples occur.

Proposition 3.5 and Lemmas 3.6 and 3.8 give a proof of Theorem 3.3. 
Remark 1. Brouwer, Cohen, and Neumaier [5, Corollary 5.8.3] use a version of Lemma 3.4 above to show that a distance-regular bipartite antipodal 2-cover of diameter 6 and valency $k \geq 3$ must be isomorphic to the 6-cube, thereby verifying their conjecture for the case of 2-covers.

Remark 2. In the proof of Lemma 3.8 the appeal to Godsil, Liebler, and Praeger's [7] is heavy-handed, as that is a long and difficult paper proving much more than needed here. Elementary arguments could be extracted from [7] to handle Lemma 3.8 directly. But that would still leave us dependent upon the classification of finite simple groups via Kantor's Theorem 3.2.

\section{References}

1. M.R. Alfuraidan, "Imprimitive distance-transitive graphs," Ph.D. Thesis, Michigan State University, March 2004.

2. M.R. Alfuraidan and J.I. Hall, "Imprimitive distance-transitive graphs with primitive core of diameter at least three," in preparation.

3. N.L. Biggs and A. Gardiner, "The classification of distance transitive graphs," unpublished manuscript, 1974.

4. J.T.M. van Bon and A.E. Brouwer, "The distance-regular antipodal covers of classical distance-regular graphs," in: Colloq. Math. Soc. Janos Bolyai, Proc. Eger 1987, 1988, pp. 141-166.

5. A.E. Brouwer, A.M. Cohen, and A. Neumaier, Distance-Regular Graphs, Springer, Berlin, 1989.

6. P.J. Cameron, Permutation Groups, London Mathematical Society Student Texts, vol. 45, Cambridge University Press, Cambridge, 1999.

7. C.D. Godsil, R.A. Liebler, and C.E. "Praeger, Antipodal distance transitive covers of complete graphs," Europ. J. Combin. 19 (1998), 455-478.

8. J. Hemmeter, "Halved graphs, Johnson and Hamming graphs," Utilitas Math. 25 (1984), 115-118.

9. J. Hemmeter, "Distance-regular graphs and halved graphs," Europ. J. Combin. 7 (1986), 119-129.

10. A.A. Ivanov, "Distance-transitive graphs and their classification," in Investigations in the Algebraic Theory of Combinatorial Objects, I.A. Faradzev, A.A. Ivanov, M.H. Klin, and A.J. Woldar (Eds.), Kluwer, Dordrecht, 1994, pp. 283-378.

11. A.A. Ivanov, R.A. Liebler, T. Penttila, and C.E. Praeger, "Antipodal distance-transitive covers of complete bipartite graphs," European J. Combin. 18 (1997), 11-33.

12. W.M. Kantor, "Classification of 2-transitive symmetric designs," Graphs Combin. 1 (1985), 165-166.

13. M.W. Liebeck, "The affine permutation groups of rank three," Proc. London Math. Soc. 54(3), (1987), 477-516.

14. D.H. Smith, "Primitive and imprimitive graphs, Quart," J. Math. Oxford 22(2), (1971), 551-557. 